\title{
Tracheal Stenosis, CTCAE
}

National Cancer Institute

\section{Source}

National Cancer Institute. Tracheal Stenosis, CT CAE. NCI Thesaurus. Code C143883.

A disorder characterized by a narrowing of the trachea. 